This item was submitted to Loughborough's Research Repository by the author.

Items in Figshare are protected by copyright, with all rights reserved, unless otherwise indicated.

\title{
Angular sway propagation in One Leg Stance and quiet stance with Inertial Measurement Units for older adults
}

PLEASE CITE THE PUBLISHED VERSION

http://dx.doi.org/10.1109/EMBC.2015.7319992

\section{PUBLISHER}

(C) IEEE

\section{VERSION}

AM (Accepted Manuscript)

\section{PUBLISHER STATEMENT}

This work is made available according to the conditions of the Creative Commons Attribution-NonCommercialNoDerivatives 4.0 International (CC BY-NC-ND 4.0) licence. Full details of this licence are available at: https://creativecommons.org/licenses/by-nc-nd/4.0/

\section{LICENCE}

CC BY-NC-ND 4.0

\section{REPOSITORY RECORD}

Kong, Weisheng, Salvatore Sessa, Di Zhang, Massimiliano Zecca, Sarah Cosentino, Hiroyuki Ishii, Daniele Magistro, Hikaru Takeuchi, Ryuta Kawashima, and Atsuo Takanishi. 2019. "Angular Sway Propagation in One Leg Stance and Quiet Stance with Inertial Measurement Units for Older Adults”. figshare. https://hdl.handle.net/2134/20537. 


\title{
Angular Sway Propagation in One Leg Stance and Quiet Stance with Inertial Measurement Units for Older Adults
}

\author{
W. Kong ${ }^{1}$, S. Sessa ${ }^{1}$, D. Zhang ${ }^{1}$, M. Zecca ${ }^{2,3}$, S. Cosentino ${ }^{1}$ \\ H. Ishii ${ }^{1}$, D. Magistro ${ }^{2}$, H. Takeuchi ${ }^{4}$, R. Kawashima ${ }^{4}$, and A. Takanishi ${ }^{5}$
}

\begin{abstract}
Postural stability degrades with age, threating the health and life quality of the older adults. One Leg Stance (OLS) is one of the standard and commonly adopted assessments for postural stability, and the postural sway in OLS has been demonstrated to be related with age. The propagation of postural sway between body segments could be a hint to the underlying mechanism of balance control. However, it is not yet fully understood. Therefore, the aim of this paper was to study the angular sways and their propagation of the head, trunk, and lower limb in healthy older adults. A cross-correlation of the normalized angular speeds was performed and the experiment with 68 older adults was conducted. The results showed that the head, hip and ankle joints affected the transfer of angular sway with a relatively lower correlation and longer latency.
\end{abstract}

\section{INTRODUCTION}

Older adults aged over 65 suffer from the decreasing postural stability [1], due to physiological disorders [2] and aging processes such as insufficient muscle strength [3], degenerated somatosensory system [4], and age-related brain changes [5].

To evaluate the postural stability, one of the standard tests used by the clinicians is One leg stance (OLS). The participants are asked to stand with only one leg and without support such as handrails. This test is popularly conducted both in clinic and community settings because it is inexpensive and time-efficient.

The most commonly used parameter for quantifying performance in OLS is time. The longest or average time for which one can stand with one leg has been demonstrated to be a useful tool for detecting balance-related disorder and frailty [6], and has been suggested to be correlated with falls [7] and decline in Activity of Daily Living (ADL) [8].

Although time measurement can be used as an assessment for postural stability, it can hardly reveal how the older adults keep postural balance and how the balance strategy is affected by aging. Therefore, sensor systems have been

*This research has been supported by the JSPS Grant-in-Aid for Young Scientists (Wakate B) [25750259] and [15K21437]. The present work was also supported in part by the Program for Leading Graduate Schools, Graduate Program for Embodiment Informatics of the Ministry of Education, Culture, Sports, Science and Technology.

${ }^{1}$ Waseda University, 2-2 Wakamatsu-cho, Shinjuku-ku, Tokyo 162-8480, Japan contactetakanishi.mech. waseda.ac.jp

${ }^{2}$ EESE, Loughborough University, Loughborough LE11 3TU, United Kingdom m. zecca@lboro.ac.uk

${ }^{3}$ National Centre for Sport and Exercise Medicine (NCSEM) England, Loughborough LE11 3TU, United Kingdom

${ }^{4}$ The Department of Functional Brain Imaging. IDAC, Tohoku University, Sendai, Japan.

${ }^{5}$ The Department of Modern Mechanical Engineering, Waseda University, and Humanoid Robotics Institute, Waseda University, Tokyo, Japan. developed to quantify (1) joint position; (2) link orientation; (3) ground reaction force; and (4) the muscle activation with cameras, inertia measurement unit (IMU), force plate, and electromyography (EMG) respectively. From the sensorbased objective measurement, Gill et al. found that the trunk postural sway significantly increased with age [9], and the increased trunk sway of the older adults was demonstrated to be related with their increased reliance on hip muscle activation [10].

Previous studies have focused mainly on the postural sway of one specific body segment and compared the sway between participants with different ages or health conditions. However, it was not full understood how the postural sway would propagate between different body segments and would be affected by joints. It is interesting to know, because the propagation of instantaneous postural sway could be a hint to the underlying dynamics and balance control. Some researchers suggested that the postural sway can be considered as a sequence of slow initial falls and noncontinuous fast adjustment impulse [11], [12]. The impulsive balance adjustment occurred suddenly in one or a number of joints and then propagate through the rest of the body. Conversely, by looking at the propagation of angular sway through the body segments, it might be possible to understand where did the balance adjustments occur. In the literature, to our best knowledge, no publication was found to study the transfer of angular impulse in different body segments.

Therefore, the aim of this work was to study the angular sway of the head, the trunk, and the lower limb to understand the mechanism that underlay the transfer of the angular impulse in healthy older adults. In this work, IMU was chosen as the measurement system, for its portability and capability of directly measuring angular velocity. In contrast, the camera-based motion capture systems, although being accurate and reliable, suffered from the errors introduced by differentiation and were limited to the laboratory setup.

\section{MATERIALS AND METHODS}

\section{A. Subject}

68 older adults ranging from 65 to 76 years old (age = $69.0 \pm 3.3$ years) were recruited (27 male, 41 female) at the Institute of Development, Aging and Cancer, Tohoku University. All participants were mentally and physically healthy (Height: $157.8 \pm 8.8 \mathrm{~cm}$; Weight: $57.4 \pm 10.4 \mathrm{~kg}$; BMI: $22.9 \pm 2.6 \mathrm{~kg} / \mathrm{m}^{2}$ ), capable of keeping static posture in quiet stance and performing locomotion tasks needed for everyday 
life. The self-reported physical exercises varied from daily jogging or strolling to no physical training.

\section{B. Equipment}

The miniaturized $(17 \times 20 \times 8.0 \mathrm{~mm} ; 3.9 \mathrm{~g})$ Inertial Measurement Unit (IMU), named WB-4R [13], was used for this study. The angular velocity, acceleration and magnetic field was measured with the triaxial MEMS-based gyroscope (LYPR540AH, $\pm 400 / \pm 1600 d p s$ ), accelerometer (LIS331DLH, $\pm 8 G$ ), and magnetometer (HMC5843, \pm 4 Gauss) respectively inside the WB-4R sensor, with sampling rate $200 \mathrm{~Hz}$. In this study only the data from gyroscope was used for data analysis, and the accelerometer data was used only for OLS phase segmentation.

Nine WB-4R IMUs were tighten on the participant's body with elastic bands, with y-axis of the sensor pointing downwards and along the link of the body (Fig. 1). Gyroscope calibration was conducted in advance to convert the raw data to the angular velocity of each body segment.

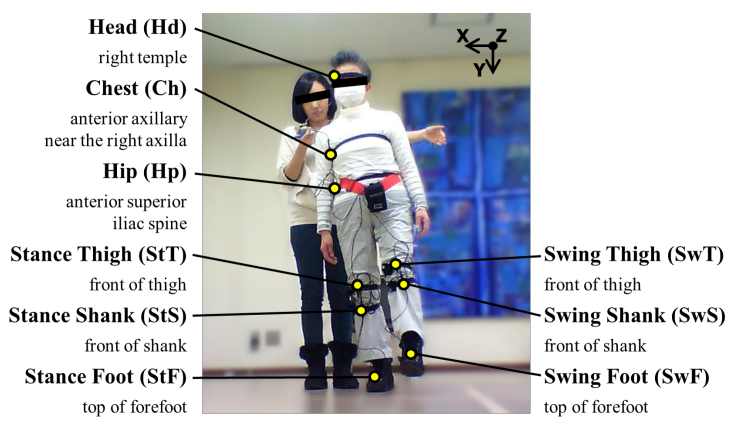

Fig. 1. Experimental setup and sensor position

\section{Experiment Protocol}

The experimental protocol started with open eyes quiet stance (QS), standing relaxed with double feet. Then the participants were asked to stand on only one leg. During OLS, the participants were required to rest their arms to side of the trunk and keep the swing leg straight. One trial was finished if the swing leg touched the ground or after standing for $30 \mathrm{~s}$. Three trials were performed for each leg starting from the left leg. A break period of one minute was given between the trials and the participants were free to interrupt the experiment whenever feeling tired. After the OLS trials, the QS was also performed one time for 10 seconds. The participants could select to perform the experiment in the most comfortable condition, wearing their shoes or removing them. The experimental protocol was approved by the ethical committees of Waseda University and Tohoku University and all participants provided written informed consent.

\section{Data Analysis}

The angular velocity from different body segments was first filtered with a 6-order Butterworth low pass filter. The cutoff frequency was set to $20 \mathrm{~Hz}$ to remove the sensor noise and eventually the artifacts, while not affecting the recording of normal human movement. The modulus of angular velocity, or angular speed, was then calculated and used as the quantification for angular sway in the following analysis. The reasons for choosing modulus instead of the value of each axis were avoiding the problem of sensor axes misalignment and easing the requirement when attaching the sensors on the subjects in clinical settings.

The data segmentation was performed to separate the one leg stance phase from the QS phase at the beginning and end of data recording. Considering the relative large data amount, an automatic segmentation was designed and applied. It was difficult to segment with only the angular velocity as the participant may take a few small steps to adjust the posture before and after the one leg stance phase. Instead, we utilized the accelerometers of the IMUs located at the thigh of both stance leg and swing leg.

The average and the standard deviation of angular speed during one leg stance phase were calculated for each body segment. These values were used to normalize the angular speed. After normalization, a cross-correlation between interconnected links (maximum latency: $\pm 500 \mathrm{~ms}$ ) was performed to find the role each joint played in one leg stance.

The exclusion criteria applied in our analysis was OLS time less than $10 \mathrm{~s}$. While it would be interesting to investigate the differences between the participants who could and those who could not successfully keep balance for $30 s$, it was not related to the aim of this paper.

\section{RESULTS}

Fig. 2 showed the averaged angular speeds during OLS (divided into left leg up (LU) and right leg up (RU)) and QS. The left column contained the upper body and stance leg, while the right column contained the swing leg. The horizontal axis of left column was reverted. Although in QS both legs were stance legs, the right leg was designated as the 'swing leg' for plotting in the same graph with OLS. The reason to plot the graph in such way was to represent the data in the similar shape of human posture in OLS and QS.
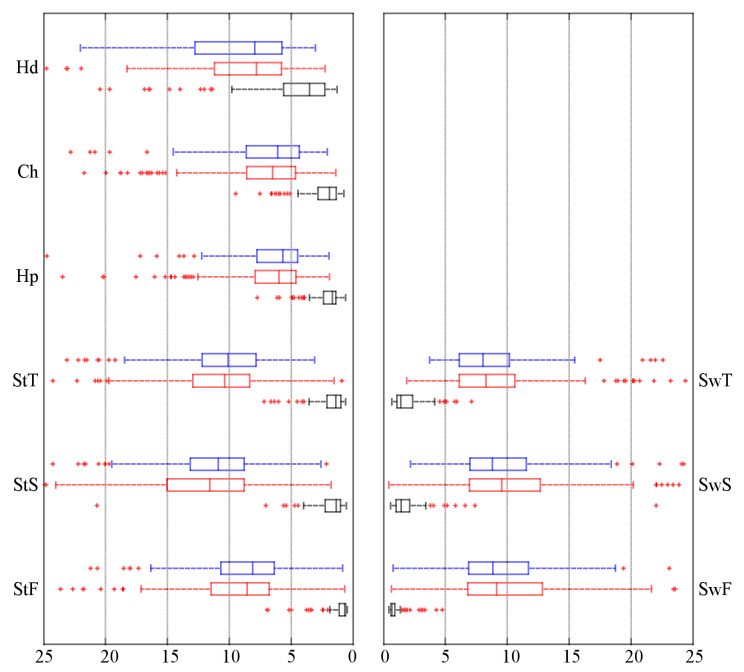

Fig. 2. Average angular speed (unit: deg/s), the blue, red and black boxes stand for left leg up(LU), right leg up(RU) and quiet stance(QS) respectively 
All of the body segments showed much larger angular sway in OLS compared with QS, especially in the lower limb. Considering the envelop of each type (left leg up, right leg up or QS), in QS the angular sway was gradually and monotonously increased from the foot to the chest, and finally dramatically increased at head. While in OLS, although the upper body (head, chest and hip) had a similar envelop with QS, the lower limb largely differed. Both legs had larger or at least comparable level of sway as the upper body. The angular sway also differed between legs. For the stance leg, the angular sway was increased from the foot to the shank, then slight decreased from the shank to the thigh. While for the swing leg, the angular speed increased monotonously from thigh to foot. In general, the largest angular sway occurred at head in QS and at stance shank in OLS. The most stable segment(s) was stance feet in QS and chest or hip in OLS. OLS showed much larger angular sway in the lower limb, and showed a rapid drop in angular speed from the lower limb to the upper body.

Table I and Fig. 3 showed the maximum value in the cross correlation between normalized angular speed of interconnected body segments, e.g. head to chest $(\mathrm{Hd} / \mathrm{Ch})$, chest to hip $(\mathrm{Ch} / \mathrm{Hp})$. OLS had much larger cross correlation values than QS, but the trend was similar. The envelop of the right column in Fig. 3 showed a wavy shape: starting from StS/StF, the correlation maximum was increased at StT/StS, then decreased at $\mathrm{Hp} / \mathrm{StT}$, increased again at $\mathrm{Ch} / \mathrm{Hp}$, and finally decreased at $\mathrm{Hd} / \mathrm{Ch}$. The envelop shape of the swing leg (left column) was similar with stance leg, but slightly higher at swing foot and swing thigh.

TABLE I

ANGULAR SPEED CORRELATION MAXIMUM VALUE

\begin{tabular}{|l||c|c||c|c|c||c|c|c|}
\hline & $\mathrm{Hd} /$ & $\mathrm{Ch} /$ & $\mathrm{Hp} /$ & $\mathrm{StT} /$ & $\mathrm{StS} /$ & $\mathrm{Hp} /$ & $\mathrm{SwT} /$ & $\mathrm{SwS} /$ \\
& $\mathrm{Ch}$ & $\mathrm{Hp}$ & $\mathrm{StT}$ & $\mathrm{StS}$ & $\mathrm{StF}$ & $\mathrm{SwT}$ & $\mathrm{SwS}$ & $\mathrm{SwF}$ \\
\hline LU & 0.62 & 0.80 & 0.47 & 0.97 & 0.80 & 0.57 & 0.91 & 0.88 \\
\hline RU & 0.58 & 0.81 & 0.49 & 0.97 & 0.77 & 0.61 & 0.92 & 0.89 \\
\hline QS & 0.30 & 0.41 & 0.27 & 0.60 & 0.19 & 0.25 & 0.55 & 0.18 \\
\hline
\end{tabular}

Table II and Fig. 4 showed the latency at which the maximum value in Fig. 3 was found. The minimum unit for latency was 5 millisecond due to sampling frequency $(200 \mathrm{~Hz})$. The positive latency means the lag and the negative means lead. In general, the higher segments follows the sway of the lower segments connected, e.g. the hip lead the chest. In Table II, in both OLS and QS the upper body had relatively larger delays among its segments $(\mathrm{Hd} / \mathrm{Ch}, \mathrm{Ch} / \mathrm{Hp})$, while the lower body had relative lower. The latency between the upper and lower body differed greatly between OLS and QS: for OLS the largest delay could be found in between the hip and stance leg $(\mathrm{Hp} / \mathrm{StT})$, but nearly zero delay between the hip and swing leg $(\mathrm{Hp} / \mathrm{SwT})$; for QS latency existed for both legs but much lower than the Hp/StT in OLS. In general, the largest latency occurred at $\mathrm{Hp} / \mathrm{StS}$ in OLS, and at $\mathrm{Hd} / \mathrm{Ch}$ in QS, while the smallest occurred between the thigh and shank (SwT/SwS, StT/StS) for both.

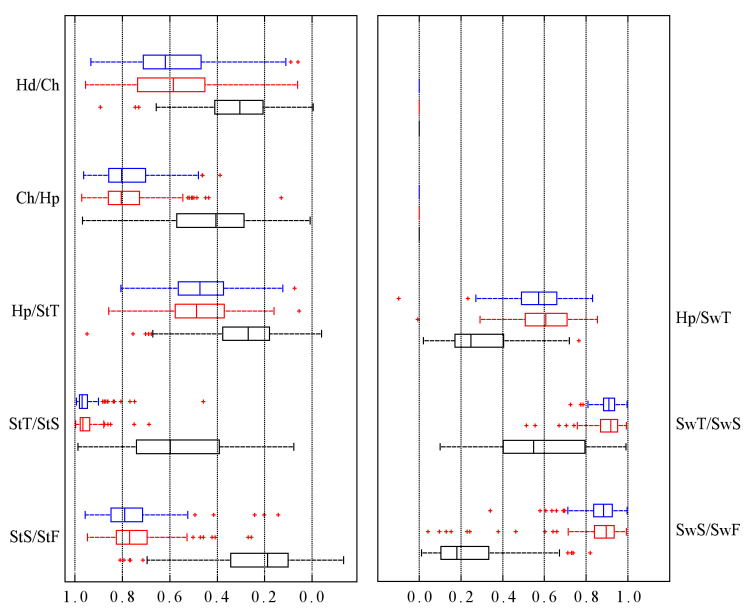

Fig. 3. Maximum of the cross correlation between magnitude of angular velocities, the blue, red and black boxes stand for left leg up(LU), right leg $\mathrm{up}(\mathrm{RU})$ and quiet stance(QS) respectively

TABLE II

ANGULAR SPEED CORRELATION LATENCY (MILLISECOND)

\begin{tabular}{|c||c|c||c|c|c||c|c|c|}
\hline & $\begin{array}{c}\mathrm{Hd} / \\
\mathrm{Ch}\end{array}$ & $\begin{array}{c}\mathrm{Ch} / \\
\mathrm{Hp}\end{array}$ & $\begin{array}{c}\mathrm{Hp} / \\
\mathrm{StT}\end{array}$ & $\begin{array}{c}\mathrm{StT} / \\
\mathrm{StS}\end{array}$ & $\begin{array}{c}\mathrm{StS} / \\
\mathrm{StF}\end{array}$ & $\begin{array}{c}\mathrm{Hp} / \\
\mathrm{SwT}\end{array}$ & $\begin{array}{c}\mathrm{SwT} / \\
\mathrm{SwS}\end{array}$ & $\begin{array}{c}\mathrm{SwS} / \\
\mathrm{SwF}\end{array}$ \\
\hline $\mathrm{LU}$ & 10 & 20 & 50 & 0 & 5 & 0 & 0 & 0 \\
\hline $\mathrm{RU}$ & 10 & 20 & 50 & 5 & 5 & 0 & 0 & 0 \\
\hline $\mathrm{QS}$ & 25 & 5 & 10 & 0 & 0 & 10 & 0 & 10 \\
\hline
\end{tabular}

\section{DISCUSSION}

Lower limbs during OLS had large angular sways which were linked with the motion of balance adjustment. The biggest component for angular sway in the stance leg was around vertical axis, which linked directly with ankle strategy. In OLS, shorter base of support in medial-lateral direction resulted in a higher difficulty to keep balance in the frontal plane. Ankle strategy actively shifted the center of pressure $(\mathrm{CoP})$ along medial-lateral direction through the inversion/eversion of the stance foot. Such rotation was generated by the small rotation in the stance shank, which was amplified and altered the rotation axis by subtalar joint [14]. Since the inversion/eversion of the foot and Tibia $\mathrm{ER} / \mathrm{IR}$ of the shank were restricted to rotate together, the latency at stance ankle was relatively low. But the correlation maximum at the ankle joint was decreased possibly by the abduction/abduction of the stance leg, contact of the foot to the floor, as well as the artifact introduced by the shoes. The swing leg, on the other hand, also had large angular sway for shifting the center of gravity $(\mathrm{CoG})$ to the center of the base of support. But different from stance ankle, the swing ankle worked passively, having a higher correlation and lower latency.

The hip joint stabilized the upper body and made the trunk the stablest link of the body, by sharply decreasing the angular sway from the stance leg (especially in the yaw direction). This could be explained by the relatively large angular inertia of upper body, the damping in hip joint, 


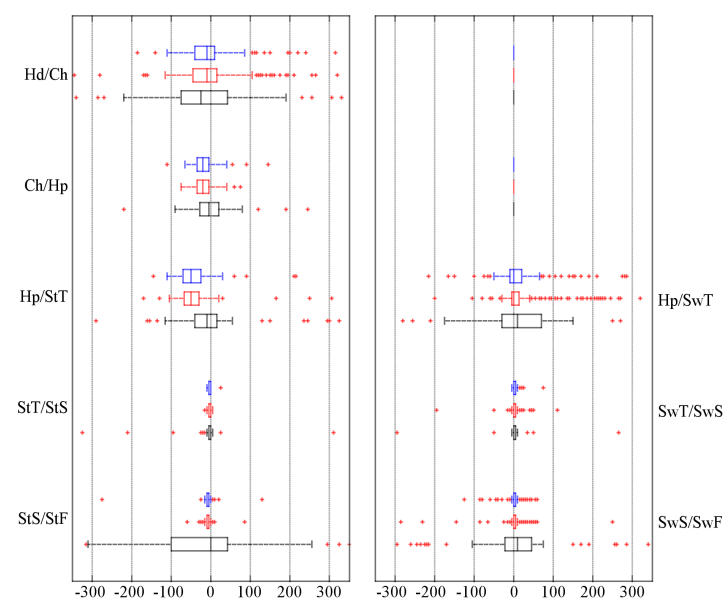

Fig. 4. Latency of the cross correlation between angular speeds (unit: $\mathrm{ms}$ ), the blue, red and black boxes stand for left leg up(LU), right leg up(RU) and quiet stance(QS) respectively

and the active stabilization control in hip joint. The latency between the angular sways of hip and stance thigh might indicate the active control in the hip joint, i.e. hip strategy. Although the relationship between latency and hip strategy was not completely clear, the fact that $\mathrm{Hp} / \mathrm{StT}$ latency was much larger in OLS than in QS seems to be consistent with the finding that reliance on hip strategy increased with difficulty of balance tasks [10]. So it would be interesting to suggest that the active control of hip joint may introduce the shift in phase.

For the upper body, the chest and hip had a higher correlation compared with other maximum values in both OLS and QS, which indicated that the angular impulse was passing through the trunk without active adjustment. The much higher latencies in OLS than in QS may reflect the change in the direction of sway. Postural sway in QS mainly oscillated in the sagittal plane while in OLS the main sway occurred in the frontal plane, resulting in different angular inertia and the muscle groups involved. The head had larger angular sway than the trunk and showed less correlation with the sway of trunk, which indicated that the head was separately controlled and actively adjusted during both OLS and QS.

To conclude, for healthy older adults the propagation of angular postural sway was affected mainly by the head, hip and stance ankle joints, showing relatively lower correlation and longer latency. This result suggested that the head, hip and stance ankle joints were actively modulated during OLS, which was consistent with the previous studies [10] which suggested high variation of joint torque in these joints. The transformation and delay in angular sway propagation may reflect the change in muscle strength and balance control strategy. In this study, only the modulus of gyroscope reading was used for data analysis except the segmentation. Therefore this method can be utilized for developing wearable, low cost, easy-to-use and objective balance evaluation system for older adults at home and clinicians at rehabilitation centers.

\section{ACKNOWLEDGMENT}

The authors would like to express their thanks to the Italian Ministry of Foreign Affairs, General Directorate for Cultural Promotion and Cooperation for its support to RoboCasa; JSPS Postdoctoral Fellowship Program for Foreigner Researchers FY2012; Tokyo Womens Medical University/Waseda University Joint Institution for Advanced Biomedical Sciences (TWIns); and International Center for Science and Engineering Programs (ICSEP) of Waseda University. The authors would also like to express their gratitude to STMicroelectronics, Life Performance Research, Okino Industries LTD, Japan ROBOTECH LTD, SolidWorks Corp, Dyden, Kyoto Kagaku Co. Ltd., and Asahi Kasei for their support to the research.

\section{REFERENCES}

[1] W. H. Organization, "WHO global report on falls prevention in older age," 2008.

[2] J. Jankovic, "Parkinsons disease: clinical features and diagnosis," Journal of Neurology, Neurosurgery \& Psychiatry, vol. 79, pp. 368376, Apr. 2008.

[3] D. Magistro, F. Candela, P. R. Brustio, M. E. Liubicich, and E. Rabaglietti, "Longitudinal Study on the Relationship Between Aerobic Endurance and Lower Body Strength in Italian Sedentary Older Adults," Journal of Aging and Physical Activity, Oct. 2014.

[4] M.-h. Hu and M. H. Woollacott, "Multisensory Training of Standing Balance in Older Adults: I. Postural Stability and One-Leg Stance Balance," Journal of Gerontology, vol. 49, pp. M52-M61, Mar. 1994.

[5] H. Baezner, C. Blahak, A. Poggesi, L. Pantoni, D. Inzitari, H. Chabriat, T. Erkinjuntti, F. Fazekas, J. M. Ferro, P. Langhorne, J. O’Brien, P. Scheltens, M. C. Visser, L. O. Wahlund, G. Waldemar, A. Wallin, M. G. Hennerici, and LADIS Study Group, "Association of gait and balance disorders with age-related white matter changes: the LADIS study," Neurology, vol. 70, pp. 935-942, Mar. 2008.

[6] T. Michikawa, Y. Nishiwaki, T. Takebayashi, and Y. Toyama, "One-leg standing test for elderly populations," Journal of Orthopaedic Science. vol. 14, pp. 675-685, Oct. 2009.

[7] "Physical Performance Measures That Predict Faller Status in Community-Dwelling Older Adults," Journal of Orthopaedic \& Sports Physical Therapy, vol. 16, pp. 123-128, Sept. 1992.

[8] M.-R. Lin, H.-F. Hwang, M.-H. Hu, H.-D. I. Wu, Y.-W. Wang, and F.C. Huang, "Psychometric comparisons of the timed up and go, one-leg stand, functional reach, and Tinetti balance measures in communitydwelling older people," Journal of the American Geriatrics Society, vol. 52, pp. 1343-1348, Aug. 2004.

[9] J. Gill, J. H. Allum, M. G. Carpenter, M. Held-Ziolkowska, A. L. Adkin, F. Honegger, and K. Pierchala, "Trunk sway measures of postural stability during clinical balance tests: effects of age," The Journals of Gerontology. Series A, Biological Sciences and Medical Sciences, vol. 56, pp. M438-447, July 2001.

[10] I. G. Amiridis, V. Hatzitaki, and F. Arabatzi, "Age-induced modifications of static postural control in humans," Neuroscience Letters, vol. 350, pp. 137-140, Oct. 2003.

[11] M. Jacono, M. Casadio, P. G. Morasso, and V. Sanguineti, "The swaydensity curve and the underlying postural stabilization process," Motor Control, vol. 8, pp. 292-311, July 2004.

[12] A. Bottaro, M. Casadio, P. G. Morasso, and V. Sanguineti, "Body sway during quiet standing: Is it the residual chattering of an intermittent stabilization process?," Human Movement Science, vol. 24, pp. 588615, Aug. 2005.

[13] W. Kong, S. Sessa, S. Cosentino, M. Zecca, K. Saito, C. Wang, U. Imtiaz, Z. Lin, L. Bartolomeo, H. Ishii, T. Ikai, and A. Takanishi, "Development of a real-time IMU-based motion capture system for gait rehabilitation," in 2013 IEEE International Conference on Robotics and Biomimetics (ROBIO), pp. 2100-2105, Dec. 2013.

[14] H. Tropp and P. Odenrick, "Postural control in single-limb stance," Journal of Orthopaedic Research, vol. 6, no. 6, pp. 833-839, 1988. 